2020". Refractive errors, is one of the priority areas for vision 2020 as they are so common and because corrective spectacles provide a remedy that is cheap, effective and associated with huge functional improvement.

Aims and Objectives To estimate the prevalence of refractive errors and colour blindness among 5-15 years school going children in Shimla.

Materials and Methods Cross sectional study carried out in the in urban and rural schools of Shimla. In a total of 2000 school children aged 5-15 years visual acuity was tested using ETDRS chart for refractive error and Ichihara colour plates for colour vision. Trained school teachers assessed visual acuity and colour vision. Students found having visual impairment by trained school teachers were further examined by refractionist.

Results Among the 2000 study subjects 956 (48\%) were males and $1044(52 \%)$ were females. The mean age of the study participants was 12.59 years $+2.25 .4 .2 \%$ were found to be having refractive error. Myopia was found to be the most prevalent type of RE followed by hypermetropia and finally astigmatism. There was no association between RE with gender. The prevalence of colour blindness was $0.3 \%$.

Conclusion Because visual impairment can have a significant impact on a child's life in terms of education and development, it is important that effective strategies be developed to eliminate this easily treated cause of visual impairment.

\section{P2-414 THE ALL IRELAND TRAVELLER BIRTH COHORT STUDY (2008-2011): EXPERIENCE FROM A NOMADIC COHORT OF FIVE HUNDRED FAMILIES}

doi:10.1136/jech.2011.142976l.44

N A Hamid, ${ }^{*}$ C Kelleher, P Fitzpatrick, For All Ireland Traveller Health Study Team. University College Dublin, Belfield, Dublin 4, Ireland

Introduction Irish Travellers are a nomadic minority group in Ireland with a strong cultural identity, who experience profound socioeconomic and health inequalities. The All Ireland Traveller Health Study is a multi-study project, one component of which is a birth cohort follow-up study. The aim of this study is to describe the methodology and findings to date.

Methodology This is a 3-year longitudinal linkage cohort study of all Irish Traveller mothers who gave birth on island of Ireland between 14 October 2008 and 13 October 2009 followed up for 1 year thereafter to assess infant mortality and health outcomes. Public Health Nurses and Traveller community health workers in Traveller Health Projects facilitated cohort ascertainment. Participating mothers carried a Parent-Held Child Record and consented to linkage to perinatal data in maternity hospitals. Infant Traveller deaths were also searched for in the General Register Office.

Results Of 987 Traveller births identified, 506 consented (51.2\%) to participation. We retrieved 491 linkage records (97\%) from the 25 maternity hospitals and 348 (68.8\%) of the Parent-Held Child Record in 37 districts. $35 \%$ of participants in the cohort still travel with $19.7 \%$ lost from follow-up as a result of nomadic practice. Preliminary follow-up data suggest infant mortality was higher in the refusal group and predominantly from congenital conditions in the perinatal period.

Conclusion The recruitment strategy was culturally appropriate and, despite challenges to engagement, longitudinal follow-up to date has been largely successful. The experience is relevant to other indigenous minority healthcare initiatives worldwide.

\section{P2-415 SMOKING-SPECIFIC COMMUNICATION AND CHILDREN'S SMOKING BEHAVIOUR: AN EXTENSION OF THE THEORY OF PLANNED BEHAVIOUR}

doi:10.1136/jech.2011.142976l.45

${ }^{1} \mathrm{M}$ Hiemstra, ${ }^{*}{ }^{1} \mathrm{R}$ Otten, ${ }^{2} \mathrm{O}$ C P van Schayck, ${ }^{1} \mathrm{R}$ C M E Engels. ${ }^{1}$ Radboud University Nijmegen, Behavioural Science Institute, Nijmegen, The Netherlands; ${ }^{2}$ Maastricht University, Care and Public health Research Institute, Maastricht, The Netherlands

Introduction Smoking starts and progresses rapidly during adolescence. Therefore, it is important to prevent youths from smoking. Previous research is mostly conducted on adolescent samples. This innovative study will focus on smoking of children aged 9-11 years old. The aim is to test whether parental smoking-specific communication and parental smoking add to smoking-specific cognitions derived from the Theory of Planned Behaviour (TPB) in predicting smoking onset.

Method A total of 1478 pairs of mothers and children participated. Structural equation models in Mplus were used to examine whether parental smoking-specific communication are important in shaping children's smoking cognitions, which affects smoking onset.

Results Pro-smoking attitude, higher frequency of communication and high parental smoking were associated with smoking onset. No significant association was found for self-efficacy, perceived norm of mother, (best) friends on smoking onset. We also found that a frequency of communication, quality of communication and parental smoking were related to smoking cognitions.

Conclusion At this age, smoking-specific communication and smoking behaviour of parents are associated with smoking cognitions. From the cognitions, only attitude is related to smoking onset. The extension of TPB is comparable with earlier research on adolescents. A difference between adolescent and child research is that cognitions have no association with smoking onset. A possible explanation can be that smoking-specific cognitions are not developed at this age because children are not engaged in smoking yet. Results can be used to develop effective family-based smoking prevention programs.

\section{P2-416 ESTIMATING THE PREVALENCE OF HEPATITIS B INFECTION AMONG PEOPLE WHO INJECT DRUGS: RESULTS FROM A GLOBAL SYSTEMATIC REVIEW}

doi:10.1136/jech.2011.142976l.46

${ }^{1} \mathrm{D}$ Horyniak, ${ }^{2} \mathrm{P}$ Nelson, ${ }^{3,4} \mathrm{~B}$ Cowie, ${ }^{5} \mathrm{H}$ Hagan, ${ }^{6} \mathrm{D} \mathrm{D}$ Jarlais, ${ }^{1} \mathrm{~L}$ Degenhardt, ${ }^{1} \mathrm{~S}$ Kinner. ${ }^{*}$ ${ }^{1}$ Burnet Institute, Melbourne, Victoria, Australia; ${ }^{2}$ National Drug and Alcohol Research Centre, University of New South Wales, Sydney, New South Wales, Australia; ${ }^{3}$ Victorian Infectious Diseases Reference Laboratory, Melbourne, Victoria, Australia; ${ }^{4}$ University of Melbourne, Melbourne, Victoria, Australia; ${ }^{5}$ New York University, New York, New York, USA; ${ }^{6}$ Chemical Dependency Institute, Beth Israel Medical Centre, New York, New York, USA

Introduction Hepatitis B virus (HBV) infection, although not as prevalent among people who inject drugs (PWID) as hepatitis C, remains a significant public health concern. We present the first detailed global review of HBV prevalence among PWID.

Methods As part of the WHO-funded Global Burden of Disease study, we undertook a systematic review of electronic databases (Medline, Embase, PsycINFO) and relevant conference abstracts to identify studies reporting HBV prevalence among PWID. Experts in the field were invited through a "viral email" to submit unpublished reports, to ensure inclusion of as many studies as possible. Studies were graded according to methodological characteristics and data quality, before national, regional and global prevalence estimates of specific HBV markers were calculated.

Results Two hundred and eighty-nine studies reporting HBV prevalence estimates from more than 50 countries were identified. Most 